\title{
Determination of the Number of Fixture Locating Points for Sheet Metal By Grey Model
}

\author{
Bo Yang , Zhongqi Wang, Yuan Yang, Zewang Jing and Yonggang Kang \\ The Ministry of Education Key Laboratory of Contemporary Design and Integrated Manufacturing Technology, Northwestern Polytechnical \\ University, No. 127, Youyi Road (West), Xi'an 710072, China
}

\begin{abstract}
In the process of the traditional fixture design for sheet metal part based on the "N-2-1" locating principle, the number of fixture locating points is determined by trial and error or the experience of the designer. To that end, a new design method based on grey theory is proposed to determine the number of sheet metal fixture locating points in this paper. Firstly, the training sample set is generated by Latin hypercube sampling (LHS) and finite element analysis (FEA). Secondly, the GM(1,1) grey model is constructed based on the established training sample set to approximate the mapping relationship between the number of fixture locating points and the concerned sheet metal maximum deformation. Thirdly, the final number of fixture locating points for sheet metal can be inversely calculated under the allowable maximum deformation. Finally, a sheet metal case is conducted and the results indicate that the proposed approach is effective and efficient in determining the number of fixture locating points for sheet metal.
\end{abstract}

\section{Introduction}

In order to not only determine the spatial location but also constrain the excessive deformation of sheet metal part, Cai et al. proposed the "N-2-1" (N>3) locating principle for sheet metal fixture design and at the same time pointed out that the "N-2-1" locating principle was more suitable for sheet metal part than "3-2-1" principle [1]. Hence, one of the keys is to find the minimum number of locators their optimal positions to keep the sheet metal part under the allowable maximum deformation and reduce the maximum sheet metal deformation as greatly as possible in fixture design based on the "N-2-1" locating principle.

To deal with the problem above, many scholars and technicians carried out lots of research. Krishnakumar et al. [2] combined genetic algorithm (GA) with finite element analysis (FEA) to search the optimum fixture layout to minimize the part deformation. Liu et al. [3] determined the initial number and positions of the locators by adding locators on the datum plane at the position with the maximum deformation repeatedly until the deformation was reduced within the range of milling accuracy. Prabhaharan et al. [4, 5] presented the fixture layout optimization methods that used GA and ant colony algorithm (ACA) separately combined with FEA to reduce the dimensional and form errors of the deformable workpiece. Through employing FEA to compute the workpiece deformation for a given fixture layout, Dou et al. [6] conducted the applications and comparisons of GA, improved GA, particle swarm optimization (PSO) and improved PSO for the fixture layout locating optimization to minimize the elastic deformation of the workpiece. Xing et al. [7] proposed a new method to optimize fixture layout by a non-domination sorting social radiation algorithm (NSSRA) combined with FEA to satisfy the requirements of the assembly tolerance. What's more, in order to reduce the computational cost and improve the optimization efficiency, approximation or surrogate models [8] such as response surface methodology (RSM), back propagation neural network $(\mathrm{BPNN})$ and radial basis function neural network (RBFNN) have been introduced and applied to computationally expensive fixture layout optimization for controlling the undesirable deformation of the flexible or deformable workpiece and improving the manufacturing accuracy in recent years [9 11]. However, most studies only optimized the sheet metal fixture locating layout for a given number of fixture locating points and few research have been carried out on the design of the number of fixture locating points.

The grey system theory was first proposed and established by Julong Deng [12, 13] in 1982 to study of the problems involving small samples and poor information. This theory states that for a grey system just some information is known and some is uncertain or fully unknown. However, there is a necessary intrinsic relation between them, through generating and exploiting the limited known information, the system behavior can be described and controlled. At present, grey system theory has been applied to predict and analyze the practical problems in different fields. Wang et al. [14] presented a systematic methodology for the thermal-error correction of a machine tool and used the grey system model to 
predict and compensate for the thermal error. Li et al. [15] studied the optimization of the thermal sensors' placement on machine tools based on grey system theory and reduced the temperature variables in the thermal error model significantly.

In this paper, a new approach based on grey system theory is presented to obtain the final number of fixture locating points for sheet metal part. With this section included, this paper is made up of six sections. The remainder of the paper is organized as follows: in section 2 , the design model of the number of fixture locating points for sheet metal part is constructed. In section 3, the bases of the GM $(1,1)$ grey model are introduced. Section 4 depicts the flowchart of the proposed method. In section 5 , a sheet metal case study is presented to verify the effectiveness and efficiency of the method. Finally, this paper is concluded in section 6.

\section{Problem formulation}

In order supply more reinforcements to avoid the excessive deformation of the workpiece at machining, assembly and measuring stages throughout the whole manufacturing process, sheet metal part is often kept under an over-constraint condition based on the so-called "N-2-1" locating principle. The principle holds that there are "N" $(\mathrm{N}>3)$ locating points on the first datum plane of sheet metal part, and "2" and "1" on the second and third datum plane respectively. Figure 1 illustrates a typical "42-1" principle, where 4 locators are required in order to support sheet metal on the primary datum plane to avoid the excessive deflection of the part. Apparently, the determination of the locator number "N", which is always more than three, is one of the main tasks to minimize the sheet metal part deformation in computer aided fixture design.

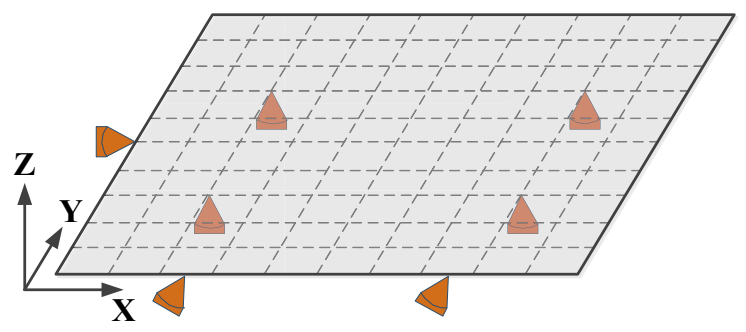

The locator on "N" The locator on " 2 " The locator on "1"

Figure 1. The "N-2-1" locating principle.

The design model for the number of fixture locating points for sheet metal part can be formulated as:

$$
\left\{\begin{array}{l}
\text { Find: } N \text { for } L=\left[l_{1}, l_{2}, \cdots, l_{N}\right] \\
F=\operatorname{Max}\left\{\varepsilon_{i}\right\}, i=1,2, \cdots, \mathrm{K} \\
\text { s.t. } \operatorname{Minimum} F \leq \delta
\end{array}\right.
$$

where $N$ is the design variable standing for the number of fixture locating points; $L=\left[l_{1}, l_{2}, \cdots, l_{N}\right]$ is the $N$ fixture locating points; $\varepsilon_{i}$ is the normal deflection of the $i$-th mesh node; $K$ is the total number of the mesh nodes of the finite element model of sheet metal part; $\delta$ is the allowable maximum deformation in engineering.

\section{Grey prediction model}

Grey means uncertain. The aim of grey system theory is to provide theory, techniques and methods for resolving (analyzing) latent and intricate grey systems which lack concerned information. As one of the most popular grey models, GM $(1,1)$ model has been studied deeply and applied in many fields since first proposed by Deng [12].

$$
\begin{aligned}
& \text { Let } X^{(0)} \text { denote the original series: } \\
& X^{(0)}=\left\{x^{(0)}(1), x^{(0)}(2), \cdots, x^{(0)}(n)\right\} \text {, where } x^{(0)}(i) \text {, } \\
& (i=1,2, \cdots, n) \text { is non-negative. }
\end{aligned}
$$

In order to reduce the noise and eliminate the disorderliness of the raw series data, the original data is converted into a monotonically increasing data series through accumulated generating operation (AGO):

$$
X^{(1)}=\left\{x^{(1)}(1), x^{(1)}(2), \cdots, x^{(1)}(n)\right\}
$$

where $x^{(1)}(k)=\sum_{i=1}^{k} x^{(0)}(i),(k=1,2, \cdots, n)$.

The new series $X^{(1)}$ is monotonic increase sequence and follows an approximate exponential law supposing the original series is smooth enough. The mean sequence can be generated by:

$$
z^{(1)}(k)=\frac{x^{(1)}(k)+x^{(1)}(k-1)}{2}, k=2,3,4 \cdots
$$

The definition type of GM $(1,1)$ model is given as:

$$
x^{(0)}(k)+a z^{(1)}(k)=b
$$

where $a$ is called the development coefficient and represents the development state of the prediction value, $b$ is called the grey action quantity and represents change contained in the data. To build the GM $(1,1)$ grey model, only a few data are needed to distinguish it. What's more, to estimate the coefficients $a$ and $b$, least square method is adopted here.

$$
\text { Let's denote } B=\left[\begin{array}{cc}
-z^{(1)}(2) & 1 \\
-z^{(1)}(3) & 1 \\
\vdots & \vdots \\
-z^{(1)}(n) & 1
\end{array}\right], \quad Y=\left[\begin{array}{c}
x^{(0)}(2) \\
x^{(0)}(3) \\
\vdots \\
x^{(0)}(n)
\end{array}\right] \text {, }
$$

$u=\left[\begin{array}{l}a \\ b\end{array}\right]$, then based on the principle of least square method, we have:

$$
\tilde{u}=\left(B^{T} B\right)^{-1} B^{T} Y
$$


Therefore, using $a$ and $b$, we can establish the following response equation of $\operatorname{GM}(1,1)$ for future prediction:

$$
\tilde{x}^{(1)}(k+1)=\left(x^{(0)}(1)-\frac{b}{a}\right) e^{-a k}+\frac{b}{a}, k=1,2, \cdots, n-1
$$

Thus the simulative value of $X^{(0)}$ can be calculated through

$$
\tilde{x}^{(0)}(k+1)=\tilde{x}^{(1)}(k+1)-\tilde{x}^{(1)}(k)
$$

At last, the $Q$ test of relative residual should be conducted on the grey model. The smaller the relative error $\mathrm{Q}$ is, the more accurate the prediction model is. The relative error is defined as:

$$
Q=\frac{1}{n} \sum_{k=1}^{n} \Delta_{k}
$$

where $\mathrm{n}$ is the total number of the original data, and $\Delta_{k}=\frac{\tilde{x}^{(0)}(k)-x^{(0)}(k)}{x^{(0)}(k)}$ is the relative error of the $k$-th original data.

\section{Method}

In this paper, a new approach to determining the number of sheet metal fixture locating points based on the "N-21 " locating principle by grey model is proposed. The flow chart shown in Fig. 2 explains the procedure of the proposed method.

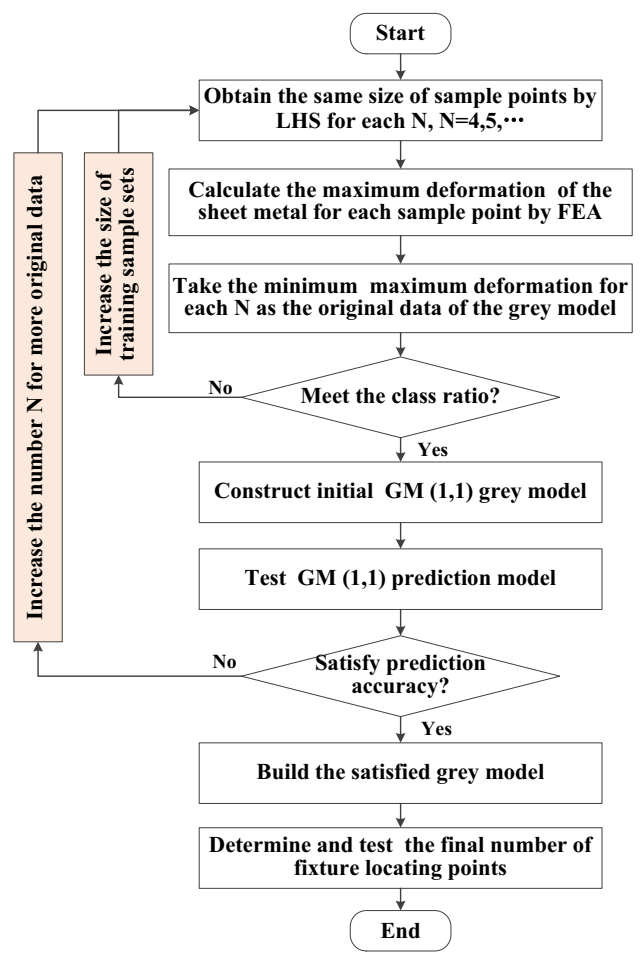

Figure 2. The flowchart of the determination of the number of fixture locating points for sheet metal by grey model.
The locator number "N" is treated as the design variable while the minimum maximum deformation of sheet metal under its dead weight is treated as the evaluation function. The finite element model of sheet metal locating is established to compute the evaluation function value, the training data set is constructed through Latin hypercube sampling (LHS) [16] and FEA and the established GM $(1,1)$ model is applied to predict the number of sheet metal fixture locating points. Taking the allowable maximum deformation as the restrictive condition, the final design variable can be inversely calculated for the appropriate maximum deformation.

\section{Case study}

In this section, the determination of the number of fixture locating points for sheet metal by grey model is demonstrated by an aluminium alloy case. The fixture locating scheme of the case based on the "N-2-1" locating principle is shown in Figure 3. The sheet metal has dimensions of $100 \mathrm{~mm} \times 100 \mathrm{~mm} \times 1 \mathrm{~mm}$ and the physical material properties are listed in Table 1 . The " $\mathrm{N}$ " locating points on the first datum plane are $l_{1}, l_{2}, \cdots, l_{\mathrm{N}}$, and the "2" locating points on the second datum plane are $F l_{1}$ and $\mathrm{Fl}_{2}$ while the "1" locating point on the third datum plane is $F l 3$. The coordinates of the fixed locating points $F l_{1}$, $\mathrm{Fl}_{2}$ and $\mathrm{Fl}_{3}$ are set as $(33,0),(67,0)$ and $(0,50)$. The locating points to be optimized are $l_{1}, l_{2}, \cdots, l_{\mathrm{N}}$ and their corresponding position coordinates are denoted by $\left(\mathrm{x}_{1}\right.$, $\left.\mathrm{y}_{1}\right),\left(\mathrm{x}_{2}, \mathrm{y}_{2}\right), \cdots,\left(\mathrm{x}_{\mathrm{N}}, \mathrm{y}_{\mathrm{N}}\right)$ respectively. In the finite element model, the grid size is set as $1 \mathrm{~mm} \times 1 \mathrm{~mm}$ and the mesh type is S4R. The allowable maximum deformation is presumably set as $\delta=0.0005 \mathrm{~mm}$.

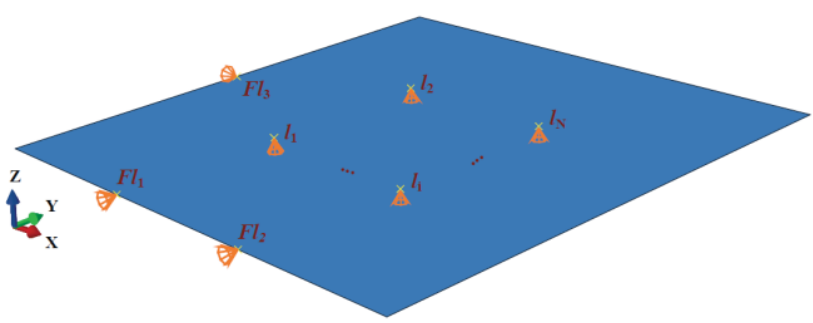

Figure 3. The initial fixture locating scheme of the aluminum alloy sheet metal part.

Table 1. The physical properties of material.

\begin{tabular}{|c|c|}
\hline & Value \\
\hline Mass density & $2.8 \times 10^{-3} \mathrm{~g} / \mathrm{mm}^{3}$ \\
\hline Young's modulus & $7.3 \times 10^{4} \mathrm{MPa}$ \\
\hline Poisson ratio & 0.33 \\
\hline
\end{tabular}

The training data set is generated by LHS and FEA. In order to calculate the evaluation values of the training data set, the commercial finite element software ABAQUS $^{\mathrm{TM}}$ [17] is employed to compute the maximum deformation of the sheet metal part under its deadweight because it is always hard or even impossible to derive the explicit and closed-form function expression between the fixture locating layout and the concerned sheet metal deformation. In our implementation, after several trials, the original data set for the grey model, as shown in 
Table 2, is obtained from eight different training data sets of the same size of 20 selected by LHS.

Table 2. The original data for GM $(1,1)$.

\begin{tabular}{|c|c|c|c|c|}
\hline $\mathrm{N}$ & 4 & 5 & 6 & 7 \\
\hline $\begin{array}{c}\mathrm{F} \\
(\mathrm{mm})\end{array}$ & $\begin{array}{l}0.001788 \\
41\end{array}$ & $\begin{array}{l}0.001595 \\
15\end{array}$ & $\begin{array}{l}0.0013054 \\
1\end{array}$ & $\begin{array}{l}0.0011525 \\
7\end{array}$ \\
\hline $\mathrm{N}$ & 8 & 9 & 10 & 11 \\
\hline $\begin{array}{c}\mathrm{F} \\
(\mathrm{mm})\end{array}$ & $\begin{array}{l}0.001046 \\
66\end{array}$ & $\begin{array}{l}0.000901 \\
08\end{array}$ & $\begin{array}{l}0.0008331 \\
11\end{array}$ & $\begin{array}{l}0.0006895 \\
32\end{array}$ \\
\hline
\end{tabular}

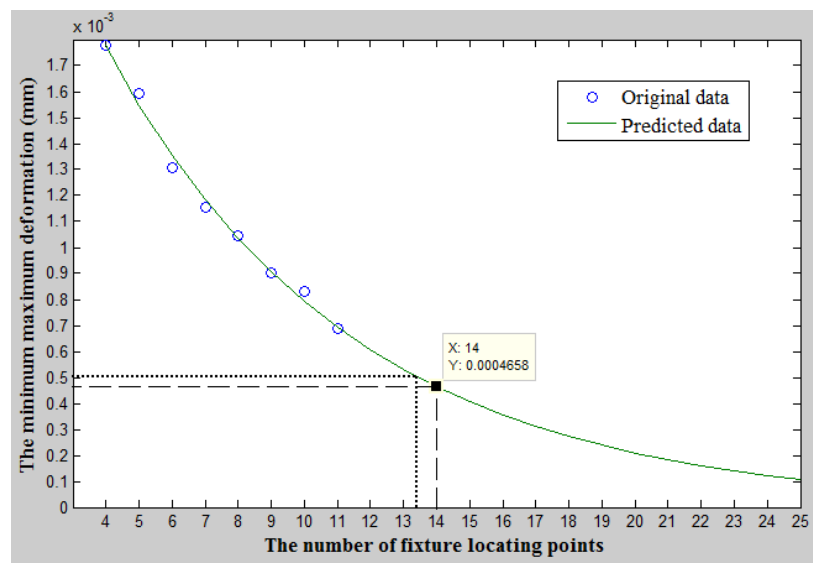

Figure 4. Prediction curve for the number of fixture locating points by grey model

In summary, referring to the flowchart of the determination of the number of fixture locating points for sheet metal by grey model, the GM $(1,1)$ grey model is established on the platform of $\operatorname{MATLAB}^{\mathrm{TM}}[18,19]$. Therefore, the prediction curve describing the mapping relationship between the number of fixture locating points and the concerned sheet metal maximum deformation can be established, as shown in Figure 4. The predicted data of the original data is listed in Table 3 and the tested data is listed in Table 4. What's more, the $\mathrm{Q}$ test of relative residuals is $0.15 \%$, and its accuracy reaches the first grade (good).

Table 3. The predicted data of the original data.

\begin{tabular}{|c|c|c|c|c|}
\hline $\mathrm{N}$ & 4 & 5 & 6 & 7 \\
\hline $\begin{array}{c}\mathrm{F} \\
(\mathrm{mm})\end{array}$ & 0.001778 & $\begin{array}{c}0.0015452 \\
6\end{array}$ & 0.0013525 & $\begin{array}{c}0.0011837 \\
8\end{array}$ \\
\hline \hline $\mathrm{N}$ & 8 & 9 & 10 & 11 \\
\hline $\begin{array}{c}\mathrm{F} \\
(\mathrm{mm})\end{array}$ & $\begin{array}{c}0.001036 \\
1\end{array}$ & $\begin{array}{c}0.0009068 \\
5\end{array}$ & $\begin{array}{c}0.0007937 \\
2\end{array}$ & $\begin{array}{c}0.0006947 \\
1\end{array}$ \\
\hline
\end{tabular}

Table 4. The predicted data for testing.

\begin{tabular}{|c|c|c|c|}
\hline $\mathrm{N}$ & 15 & 20 & 25 \\
\hline $\begin{array}{c}\text { F by grey } \\
\text { model } \\
(\mathrm{mm})\end{array}$ & 0.0004077 & 0.00020941 & 0.00010756 \\
\hline $\begin{array}{c}\text { F by FEA } \\
(\mathrm{mm})\end{array}$ & 0.000416886 & 0.00020959 & 0.000107342 \\
\hline $\begin{array}{c}\text { Relative } \\
\text { error }\end{array}$ & $2.20 \%$ & $0.09 \%$ & $0.20 \%$ \\
\hline
\end{tabular}

Referring to the prediction curve, the final number of fixture locating points and the corresponding maximum deformation by GM $(1,1)$ are shown in Table 5 . For further comparative analysis, the maximum deformation of the sheet metal part with the final number of fixture locating points of 14 is also calculated by FEA. The results are also listed in Table 5 . It can be seen from the table that the result obtained by the proposed approach shows fine agreements with that by FEA and that the final relative error $(0.21 \%)$ is within the precision requirement $(5 \%)$.

Table 5. The relative errors of the prediction model.

\begin{tabular}{|c|c|c|}
\hline $\begin{array}{c}\text { F by grey model } \\
(\mathrm{mm})\end{array}$ & $\begin{array}{c}\text { F by FEA } \\
(\mathrm{mm})\end{array}$ & The relative error \\
\hline 0.000466 & 0.000467 & $0.21 \%$ \\
\hline
\end{tabular}

\section{Conclusion}

In order to inversely determine of the minimum number of fixture locating points for sheet metal part under the allowable maximum deformation, this paper establishes a GM $(1,1)$ based grey prediction model to describe the mapping relationship between the number of fixture locating points and the corresponding sheet metal maximum deformation. The major contributions of this paper include:

(1) A prediction method based on $\operatorname{GM}(1,1)$ grey model for the number of sheet metal fixture locating points is developed, and the prediction precision meets the general requirement at the industry level.

(2) After the determination of the final number of sheet metal fixture locating points, the subsequent sheet metal fixture locating layout design and optimization can be conducted. In addition, the approach by grey prediction model can be further extended and applied to the fixture design subject to more complex loading conditions.

\section{Acknowledgements}

This work has been supported by National Natural Science Foundation of China (Grant No. 51375396).

\section{References}

1. W. Cai, S. J. Hu, J. X. Yuan, Deformable Sheet Metal Fixturing: Principles, Algorithms, and Simulations. ASME J. Manuf. Sci. Eng., 118, 3:318324 (1996)

2. K. Krishnakumar, S. N. Melkote, Machining fixture layout optimization using the genetic algorithm. Int. J. Mach. Tools Manuf., 40, 4:579-598 (2000)

3. S. G. Liu, L. Zheng, Z. H. Zhang, Z. Z. Li, D. C. Liu, Optimization of the number and positions of fixture locators in the peripheral milling of a low-rigidity workpiece. Int. J. Adv. Manuf. Technol., 33, 7-8: 668-676 (2007)

4. G. Prabhaharan, K. P. Padmanaban, R. Krishnakumar, Machining fixture layout 
optimization using FEM and evolutionary techniques. Int. J. Adv. Manuf. Technol., 32, 11-12:1090-1103 (2007)

5. K. P. Padmanaban, K. P. Arulshri, G. Prabhakaran, Machining fixture layout design using ant colony algorithm based continuous optimization method. Int. J. Adv. Manuf. Technol., 45, 9:922-934 (2009)

6. J. P. Dou, X. S. Wang, L. Wang, Machining fixture layout optimisation under dynamic conditions based on evolutionary techniques. Int. J. Prod. Res., 50, 15:4294-4315 (2012)

7. Y. F. Xing, M. Hu, H. Zeng, Y. S. Wang, Fixture layout optimisation based on a non-domination sorting social radiation algorithm for auto-body parts. Int. J. Prod. Res., 53, 11:3475-3490 (2015)

8. T. W. Simpson, A. J. Booker, D. Ghosh, A. A. Giunta, P. N. Koch, R. J. Yang, Approximation methods in multidisciplinary analysis and optimization: a panel discussion. Struct. Multidiscip. O., 27, 5:302-313 (2004)

9. M. Hamedi, Intelligent fixture design through a hybrid system of artificial neural network and genetic algorithm. Artif. Intell. Rev., 23, 3: 295-311 (2005)

10. C. Lu, H. W. Zhao, Fixture layout optimization for deformable sheet metal workpiece. Int. J. Adv. Manuf. Technol., 78, 1-4: 85-98 (2015)

11. Z. Q. Wang, B. Yang, Y. G. Kang, Y. Yang, Development of a prediction model based on RBF neural network for sheet metal fixture locating layout design and optimization, Computational Intelligence and Neuroscience, 2016: 1-6 (2016)

12. J. L. Deng, Introduction to grey system theory. J. Grey Syst., 1, 1: 1-24 (1989)

13. J. L. Deng, Grey system theory, Huazhong University of Science and Technology Press, Wuhan, (2002) (in Chinese)

14. Y. Wang, G. Zhang, K. S. Moon, J. W. Sutherland, Compensation for the thermal error of a multi-axis machining center. J. Mater. Process. Tech, 75, 1: 4553 (1998)

15. Y. X. Li, J. G. Yang, T. Gelvis, Y. Y. Li, Optimization of measuring points for machine tool thermal error based on grey system theory. Int. J. Adv. Manuf. Technol., 35, 7-8: 745-750 (2008)

16. M. D. Mckay, R. J. Beckman, W. J. Conover, A comparison of three methods for selecting values of input variables in the analysis of output form a computer code. Technometrics, 42, 1: 55-61 (2000)

17. D. S. Simulia, Abaqus 6.12 documentation, Providence, Rhode Island, US, (2012)

18. J. W. Zhuo, Application of MATLAB Software in Mathematical Modeling, Beihang University PRESS, Beijing, (2011) (in Chinese)

19. Matlab 8.0, http://www.mathworks.com/, (2012) 